\title{
Gastrointestinal Symptoms in Autism Spectrum Disorders
}

\author{
Jenna Logan Schuppener and Jennifer Harrison Elder \\ University of Florida
}

Faculty mentor: Jennifer Harrison Elder, College of Nursing

\begin{abstract}
The purpose of this study was to characterize individuals with autism spectrum disorders (ASD), who are part of a larger ASD study, in order to determine clinically useful ways to evaluate gastrointestinal (GI) symptomology. Thirty-three children (ages 3-16) were recruited from a variety of community agencies. Potential participants were screened using the Autism Diagnostic Observation Schedule (ADOS). Parental informed consent was obtained from all participants and child assent when appropriate. Parents completed the Gastrointestinal Symptom Rating Scale, Bristol Stool Chart (BSC), and sociodemographic form. Of the 33 participants, 14 were currently experiencing gastrointestinal symptoms and had abnormal bowel movements. Seven had a history of gastrointestinal symptoms and abnormal bowel movements; two parents were unsure of their gastrointestinal health. According to the BSC rescore, 13 participants reported hard stools, 14 had normal stools, and six had loose stools. Moderate correlations were found among many GI symptoms $\left(\mathrm{r}_{33}=.396-.751\right)$ indicating that these could be clustered to create a profile. These findings are clinically significant because GI symptoms in ASD are difficult to diagnose due to communication and social impairments and may go undiagnosed and untreated. Further characterizing GI symptomology in this population is a logical role for nurse researchers since nurses are often on the front line intervening within the ASD population.

Keywords: autism spectrum disorder, gastrointestinal symptomatology, gut-brain connection
\end{abstract}

\section{Introduction}

\section{Definition of Autism}

Autism spectrum disorders (ASD) are neurodevelopmental disorders where individuals exhibit impairments with social interactions and communication. They often have narrow scope of interests as well as stereotyped behavior represented by repetitive patterns in speech or movement (Center for Disease Control and Prevention, 2018). Intellectual abilities, including reasoning and problem solving, in ASD range from savant to profoundly challenged. Depending on where individuals fall on the spectrum, they require varying amounts of assistance with activities of daily living.

The Center for Disease Control estimates the prevalence of ASD is 1 in every 59 children. The statistic represents a 15 percent increase from the previous estimate (Center for Disease Control 
and Prevention, 2018). ASD is prevalent across all races, ethnicities, and socioeconomic levels. However, ASD occurs four times more often in males than females. Currently, there are no laboratory tests to diagnose ASD. Rather, diagnosis is based on the child's development and behavioral manifestations. Some theorized risk factors include specific genes (Huquet, Ey, \& Bourgeron, 2013), sibling with ASD (Ozonoff et al., 2011), chromosomal or genetic conditions (DiGuiseppi et al., 2010) (Hall, Lightbody, \& Reiss, 2008), and children of advanced aged parents (Durkin et al., 2008).

Although the multifactorial origin of ASD is now evident, in 1998 many people were deceived into believing that vaccines produced gastrointestinal (GI) pathology leading to ASD. Dr. Andrew Wakefield alleged that the measles-mumps-rubella vaccine produced "autistic enterocolitis" followed by developmental regression (Wakefield et al., 1998). Despite the faulty research practices, the study has had a transcendental impact. Measles was nearly eradicated, but as a result of Wakefield's research many parents do not vaccinate their children leading to an increase in preventable diseases. Unfortunately, the cause of gastrointestinal pathology in individuals with ASD remains unknown. Proposed theories include gluten sensitivity (Lau et al., 2013) and lactase deficiency (Williams et al., 2011).

\section{Presence of GI Symptoms}

Valicenti-Mcdermott et al. (2006) analyzed the frequency of gastrointestinal symptoms in children with ASD. Out of the 50 participants in the ASD cohort 70\% presented with GI symptoms compared to only $28 \%$ of neurotypical children. Commonly reported symptoms included abnormal stool pattern, recurrent constipation, vomiting, belching, gaseousness, abdominal distension and abdominal pain (Valicenti-Mcdermott et al., 2006). The Fulceri et al. (2016) study further highlights the prevalence of GI symptomatology in the ASD population compared to neurotypical counterparts. One out of three children displayed at least one of the measured GI symptoms when compared to neurotypical participants. These symptoms included constipation, diarrhea, nausea, painful bowel movements, stomach aches, vomiting, and absence of eating. Constipation and abdominal pain were ubiquitous symptoms in the ASD cohort (Fulceri et al, 2016). Chandler et al.'s (2013) parent-reported gastrointestinal symptoms revealed increased constipation in the ASD cohort compared to the neurotypical and special educational needs cohort. Moreover, the investigators identified increased occurrence of vomiting and diarrhea in the ASD cohort compared to the neurotypical cohort and increased abdominal pain in 
the ASD cohort than the special education needs cohort. Bresnahan's et al. (2015) prospective cohort study $(n=114,516)$ had increased parental reports of constipation and diarrhea in children with ASD compared to typically developing individuals. Chaidez, Hansen, \& Hertz-Picciotto (2013) randomize control trial had similar findings to those of Bresnahan's et al. (2015). They identified that diarrhea and constipation affect children with ASD and developmental disorders three times more than in typically developing children (Chaidez et al., 2013).

Despite the evidence concerning the prevalence of GI symptoms in ASD, many primary care providers routinely fail to ask about GI symptoms (Buie et al., 2010). As a result, the symptoms are unrecognized and untreated. Feasibly, the main reason these symptoms are unaddressed is due to one of the cardinal features of ASD, communication impairment.

Fulceri et al. (2016) found that GI symptoms in individuals with ASD correlated with increased behavioral problems, including tantrums, anxiety, and oppositional defiant behaviors. Gorrindo's et al. (2012) research on ASD and GI manifestations provided evidence to support the correlation between constipation and language impairment. A common coping mechanism for this population is self-injurious, hyperactive, or stereotyped behavior (Chaidez et al., 2013). Researchers conducting a case study analyzed the history of a 5-year-old nonverbal boy with ASD, significant constipation, and self-injurious behavior (Schweitzer, James, Jenkins, Reiff, \& Stein, 2016). After the constipation was treated, the acute self-injurious behavior ceased. The lack of attention to GI symptoms has the potential to lead to detrimental coping techniques.

\section{The Gut Brain Connection and Gut Microbiome in ASD Populations}

Arguably, the most prominent theory related to the cause of ASD is the gut-brain axis. The gut-brain axis links cognition and emotion in the brain with intestinal function. Research suggests a link between gut microbiota and the brain in individuals with ASD (Carbotti, Scirocco, Maselli, \& Severi, 2015). The current working hypothesis is that aberrant microbiota catalyzes an immune response in the GI mucosa that increases permeability in the cells, activates sensory pathways resulting in increased epithelial permeability, activates nociceptive pathways and impairs physiologic regulation of the enteric nervous system (Simrén et al., 2012).

The microbiome in children with ASD differs from neurotypical children. Children with ASD and abdominal pain displayed IL 6 and tryptophan. However, children with ASD and functional GI disorders mucosal biopsy displayed serotonergic metabolites and inflammatory cytokines (Luna et al., 2017). Srikantha \& Mohajeri (2018) compared the gut microbiome of 
neurotypical and individuals with ASD and identified increased Firmicutes to Bacteroidetes ratio. In the ASD cohort, the decreased Bacteriodetes include Alistipes, Bilophila, Dialister, Parabacteroides, and Veillonella. The Bacteriodetes Collinsella, Corynebacterium, Dorea, and Lactobacillus were significantly increased. The bacteria in neurotypical and ASD cohorts with constipation differed with increased levels of Escherichia/Shigella and Clostridium cluster XVIII (Strati et al., 2017). However, the severity of the ASD phenotypes had no impact on the bacterial community.

The microbiota in the gastrointestinal mucosa have the potential to produce noxious metabolites that travel to the central nervous system (Altieri et al., 2011). Altieri et al. (2011) found that increased intestinal penetrability leads to an increase in toxic metabolites in the central nervous system. In an experimental study on mice, propionic acid was injected into the cerebrum the mice became socially impaired. The bacterial product of $\mathrm{p}$-cresol is propionic acid. Increased levels of the urinary biomarker p-cresol is associated with increased severity of autism in males. The variation in gut microbiota indicates a possible avenue to target for relief of GI symptoms.

Based on the literature reviewed, there appears to be sufficient background suggesting that more work is needed in the area of GI symptomatology and ASD. Thus, the purpose of this current study was to identify GI symptoms that routinely occur together in the ASD population.

\section{Methodology}

\section{Participants}

As shown in Table 1, the participants included females and males ranging from 3-16 years of age. Participants were recruited from UF Health Shands Hospital, Behavioral Analysis Support Systems, Florida Autism Center, Lamp Post, Fundamental Therapy Solutions, Launchpad Therapy, Worthington Pediatrics, and Center for Autism and Related Disorders. Organizations were contacted and approved for flyer distribution. Dr. Jennifer Elder, co-investigator, confirmed that participants met the DSM V criteria through ADOS administration. Exclusion criteria prohibited individuals with genetic disorders contributing to ASD from participating in the study. 
Table 1

Demographics of study sample.

\begin{tabular}{lccc}
\hline Characteristic & Total $(\mathrm{N}=33)$ & Male $(\mathrm{N}=25)$ & Female $(\mathrm{N}=8)$ \\
\hline Age in years M (SD) & $8.39(3.50)$ & $8.24(3.36)$ & $8.88(4.12)$ \\
aADOS score M (SD) & $22.17(9.11)$ & $23.67(9.02)$ & $21.33(9.51)$ \\
Module 1 n (\%) & $12(37.5)$ & $10(31.25)$ & $2(6.25)$ \\
Module 2 n (\%) & $9(28.125)$ & $6(18.75)$ & $3(9.38)$ \\
Module 3 n (\%) & $10(31.25)$ & $7(21.88)$ & $3(9.38)$ \\
Module 4 n (\%) & $1(3.125)$ & $1(3.125)$ & $0(0)$ \\
Ethnic Group & $23(69.70)$ & $17(68.00)$ & $6(75.00)$ \\
White, non-Hispanic n (\%) & $1(3.03)$ & $1(4.00)$ & $0(0.00)$ \\
Black, non-Hispanic n (\%) & $3(6.06)$ & $2(8.00)$ & $1(12.50)$ \\
Hispanic n (\%) & $1(3.03)$ & $1(4.00)$ & $0(0.00)$ \\
American Indian, Eskimo, or & & & \\
Aleutian n (\%) & $0(0)$ & $0(0.00)$ & $0(0.00)$ \\
Asian-Pacific Islander n (\%) & $5(15.15)$ & $4(16.00)$ & $1(12.50)$ \\
Other n (\%) & &
\end{tabular}

Note. ${ }^{\mathrm{a}} \mathrm{N}=32$, missing one male

*Assessment Tools (see Appendix A for additional information)

\section{Employing the Gastrointestinal Symptom Rating Scale (GSRS) in ASD. The GSRS is an}

interview-based scale comprised of fifteen questions for assessing gastrointestinal symptoms in individuals with irritable bowel syndrome and peptic ulcer disease. The GSRS provides a multidimensional approach to assessment by including intensity, frequency, duration, and impact on activities of daily living (McMillan, 2001). The scale measures abdominal pain, acid regurgitation, nausea and vomiting, abdominal distension, eructation (act of belching) (Eructation. (n.d.), increased flatus, increased passage of stools, heartburn, sucking sensations in the epigastrium, borborygmus (intestinal rumbling caused by moving gas) (Borborygmus, n.d.), loose stools, hard stools, urgent need for defecation, and feeling of incomplete evacuation. The symptoms are followed by four descriptions of increasing intensity spanning from zero to three. Zero indicates absence of symptoms, whereas three indicates severe symptomology.

Employing the Bristol Stool Chart (BSC) in ASD. The BSC contains seven images of stool with corresponding descriptors. BSC is widely used to measure varying levels of constipation and diarrhea (1=very hard, $7=$ liquid).

Autism Diagnostic Observational Schedule (ADOS)-1 and ADOS-2. The ADOS is considered to be a gold standard assessment tool for individuals with ASD. The tool is used to confirm ASD diagnose for individuals with a variety of ages, developmental skills, and 
communication abilities (Mccrimmon \& Rostad, 2013). The ADOS is scored in five main categories including communication, reciprocal social interaction, communication and social interaction total, imagination and creativity, and stereotyped and restricted behaviors.

\section{Procedures}

The purpose and procedures were clearly outlined in the informed consent packet provided and explained preceding an interview with the principal investigator, Dr. Elder. After the informed consent was signed, the parents answered the questions in the sociodemographic form, Child Sensory Profile, Social Responsiveness Scale, and Behavior Rating Inventory of Executive Function 2. As the parents were assisted with accurately finishing the forms, Dr. Elder conducted the ADOS-1 with each child. Prior to initiating the test, the principal investigator assessed the participants' verbal skills to ensure the correct ADOS module was used. During the evaluation parents were asked twelve questions related to their child's gastrointestinal health and rated their stool utilizing the BSC.

\section{Results}

Of the 33 participants, 14 participants were currently experiencing GI symptoms and abnormal bowel movements. Seven had a history of GI symptoms and abnormal bowel movements, two parents stated that they were unsure if their children experience GI symptoms or have abnormal bowel movements.

According to the BSC Rescore, 13 participants reported having hard stools, 14 had normal stools, and six had loose stools. The BSC was rescored to represent the categories of stool. Soft stools are represented by types 1 and 2, normal consistency stools are types 3 and 4 , and hard stools are types 5, 6, and 7. Spearman's rho statistical test was utilized to evaluate the correlations of the various GI symptoms (see Appendix B).

\section{Discussion}

\section{Major Findings}

The most significant findings were the strong correlations found between nausea and vomiting with abdominal distension $\left(\mathrm{r}_{33}=.751, \mathrm{p}=.000\right)$ and feeling of incomplete evacuation $\left(\mathrm{r}_{33}=.636\right.$, $\mathrm{p}=.000$ ). Overall, it is important to acknowledge the many gastrointestinal symptoms that occur in the ASD population. It is likely that an individual presenting with one symptom may have 
several other pervasive symptoms. Therefore, it is vital to understand all possible underlying and correlating symptomatology to ensure best patient care.

\section{Clinical Significance}

GI symptoms are pervasive within the ASD community and difficult to diagnose due to varying levels of communicative abilities and clinical presentations. Therefore, many GI symptoms go undiagnosed and untreated. The clustering of GI symptoms can be utilized to target clinician's assessment to the correlating symptoms. For example, if a child with ASD presents with recurrent nausea and vomiting, health care providers can refer to the correlation matrix to discover strong and moderate correlating symptoms including incomplete evacuation, abdominal distension, and acid regurgitation. Although the client's chief complaint may not be the correlating symptoms, the additional information provides the opportunity to treat the correlating symptoms and ask further questions about past GI health. Understanding frequently occurring symptoms not only gives providers a more accurate clinical representation of their patient, but also gives the provider the chance to better care for patients. This gives health care providers the opportunity to delve deeper into their assessment and identify correlating symptoms. It is likely that the correlating symptoms are often contributing to the chief complaint. By addressing the underlying symptoms clinicians may be able to minimize or eliminate the primary symptom.

A focused assessment based on the correlating symptomatology has the potential to treat GI symptoms that may be unrecognized otherwise. Caring for these symptoms has implications greater than simply addressing the symptoms. Gastrointestinal pathology has been linked to aberrant behaviors in ASD. Counseling parents of ASD children regarding potential symptomology may lead to earlier detection to prevent further discomfort from painful symptoms and other complications such as the children in the anecdotal reports.

\section{Implications for Future Research}

Although this research was a pilot study with a small sample size and relied heavily on parent report, it highlights the importance of further research on the gut-brain connection in autism. Needed is more research using more comprehensive tools, such as the Rome Criteria (Valicenti-Mcdermott et al., 2016). The results indicate the need for an ASD specific GI assessment. Once a systematic assessment tool is designed, researchers can obtain more information to recommend treatment options for GI symptoms and have a better understanding of the effects of interventions on ASD behavior. 


\section{References}

Altieri, L., Neri, C., Sacco, R., Curatolo, P., Benvenuto, A., Muratori, F., Persico, A. M. (2011). Urinaryp-cresol is elevated in small children with severe autism spectrum disorder. Biomarkers, 16(3), 252-260. doi:10.3109/1354750x.2010.548010

Blake, M. R., Raker, J. M., \& Whelan, K. (2016). Validity and reliability of the Bristol Stool Form Scale in healthy adults and patients with diarrhoea-predominant irritable bowel syndrome. Alimentary Pharmacology \& Therapeutics,44(7), 693-703. doi:10.1111/apt.13746

Borborygmus. (n.d.). Retrieved February 3, 2019, from https://www.merriamwebster.com/dictionary/borborygmus

Bresnahan, M., Hornig, M., Schultz, A. F., Gunnes, N., Hirtz, D., Lie, K. K., Lipkin, W. I. (2015). Association of Maternal Report of Infant and Toddler Gastrointestinal Symptoms With Autism. JAMA Psychiatry,72(5), 466. doi:10.1001/jamapsychiatry.2014.3034

Buie, T., Campbell, D. B., Fuchs, G. J., Furuta, G. T., Levy, J., Vandewater, J., Winter, H. (2010). Evaluation, Diagnosis, and Treatment of Gastrointestinal Disorders in Individuals With ASDs: A Consensus Report. Pediatrics, 125(Supplement 1). doi:10.1542/peds.2009-1878c

Carbotti, M., Scirocco, A., Maselli, M. A., \& Severi, C. (2015). The gut-brain axis: Interactions between enteric microbiota, central and enteric nervous systems. Annals of Gastroenterology,203-209. Retrieved March 19, 2019.

Centers for Disease Control and Prevention. (2018). Autism Spectrum Disorder (ASD). Retrieved September 7, 2018, from https://www.cdc.gov/ncbddd/autism/data.html

Chaidez, V., Hansen, R. L., \& Hertz-Picciotto, I. (2013). Gastrointestinal Problems in Children with Autism, Developmental Delays or Typical Development. Journal of Autism and Developmental Disorders,44(5), 1117-1127. doi:10.1007/s10803-013-1973-x

Chandler, S., Carcani-Rathwell, I., Charman, T., Pickles, A., Loucas, T., Meldrum, D., Baird, G. (2013). Parent-Reported Gastro-intestinal Symptoms in Children with Autism Spectrum Disorders. Journal of Autism and Developmental Disorders,43(12), 2737-2747. doi:10.1007/s10803-013-1768-0

DeBildt, A., Sytema, S., Ketelaars, C., Kraijer, D., Mulder, E., Volkmar, F., \& Minderaa, R. (2004). Interrelationship Between Autism Diagnostic Observation Schedule-Generic (ADOS-G), Autism Diagnostic Interview-Revised (ADI-R), and the Diagnostic and Statistical Manual of Mental Disorders (DSM-IV-TR) Classification in Children and Adolescents with Mental Retardation. Journal of Autism and Developmental Disorders,34(2), 129-137. doi:10.1023/b:jadd.0000022604.22374.5f

Diguiseppi, C., Hepburn, S., Davis, J. M., Fidler, D. J., Hartway, S., Lee, N. R., Robinson, C. (2010). Screening for Autism Spectrum Disorders in Children With Down Syndrome. Journal of Developmental \& Behavioral Pediatrics,31(3), 181-191. doi:10.1097/dbp.0b013e3181d5aa6d 
Durkin, M. S., Maenner, M. J., Newschaffer, C. J., Lee, L., Cunniff, C. M., Daniels, J. L., Schieve, L. A. (2008). Advanced Parental Age and the Risk of Autism Spectrum Disorder. American Journal of Epidemiology,168(11), 1268-1276. doi:10.1093/aje/kwn250

Eructation. (n.d.). Retrieved February 3, 2019, from https://www.merriamwebster.com/dictionary/eructation

Fulceri, F., Morelli, M., Santocchi, E., Cena, H., Bianco, T. D., Narzisi, A., Muratori, F. (2016). Gastrointestinal symptoms and behavioral problems in preschoolers with Autism Spectrum Disorder. Digestive and Liver Disease,48(3), 248-254. doi:10.1016/j.dld.2015.11.026

Gorrindo, P., Williams, K. C., Lee, E. B., Walker, L. S., Mcgrew, S. G., \& Levitt, P. (2012). Gastrointestinal Dysfunction in Autism: Parental Report, Clinical Evaluation, and Associated Factors. Autism Research,5(2), 101-108. doi:10.1002/aur.237

Hall, S. S., Lightbody, A. A., \& Reiss, A. L. (2008). Compulsive, Self-Injurious, and Autistic Behavior in Children and Adolescents With Fragile X Syndrome. American Journal on Mental Retardation,113(1), 44. doi:10.1352/0895-8017(2008)113[44:csaabi]2.0.co;2

Huguet, G., Ey, E., \& Bourgeron, T. (2013). The Genetic Landscapes of Autism Spectrum Disorders. Annual Review of Genomics and Human Genetics, 14(1), 191-213. doi:10.1146/annurev-genom-091212-153431

Kamp-Becker, I., Albertowski, K., Becker, J., Ghahreman, M., Langmann, A., Mingebach, T., . . . Stroth, S. (2018). Diagnostic accuracy of the ADOS and ADOS-2 in clinical practice. European Child \& Adolescent Psychiatry,27(9), 1193-1207. doi:10.1007/s00787$018-1143-\mathrm{y}$

Kang, D., Adams, J. B., Gregory, A. C., Borody, T., Chittick, L., Fasano, A., Krajmalnik-Brown, R. (2017). Microbiota Transfer Therapy alters gut ecosystem and improves gastrointestinal and autism symptoms: An open-label study. Microbiome,5(1). doi:10.1186/s40168-016-0225-7

Kulich, K. R., Madisch, A., Pacini, F., Piqué, J. M., Regula, J., Rensburg, C. J., Wiklund, I. K. (2008). Reliability and validity of the Gastrointestinal Symptom Rating Scale (GSRS) and Quality of Life in Reflux and Dyspepsia (QOLRAD) questionnaire in dyspepsia: A six-country study. Health and Quality of Life Outcomes, 6(1), 12. doi:10.1186/1477-7525-6-12

Lau, N. M., Green, P. H., Taylor, A. K., Hellberg, D., Ajamian, M., Tan, C. Z., Alaedini, A. (2013). Markers of Celiac Disease and Gluten Sensitivity in Children with Autism. PLoS ONE,8(6). doi:10.1371/journal.pone.0066155

Luna, R. A., Oezguen, N., Balderas, M., Venkatachalam, A., Runge, J. K., Versalovic, J., Williams, K. C. (2017). Distinct Microbiome-Neuroimmune Signatures Correlate With Functional Abdominal Pain in Children With Autism Spectrum Disorder. Cellular and Molecular Gastroenterology and Hepatology,3(2), 218-230. doi:10.1016/j.jcmgh.2016.11.008

Mccrimmon, A., \& Rostad, K. (2013). Test Review: Autism Diagnostic Observation Schedule, Second Edition (ADOS-2) Manual (Part II): Toddler Module. Journal of Psychoeducational Assessment,32(1), 88-92. doi:10.1177/0734282913490916 
McMillin, D. (2001, October). Headache Research Report. Retrieved January 27, 2019, from http://www.meridianinstitute.com/reports/headache.html

Ozonoff, S., Young, G. S., Carter, A., Messinger, D., Yirmiya, N., Zwaigenbaum, L., Stone, W. L. (2011). Recurrence Risk for Autism Spectrum Disorders: A Baby Siblings Research Consortium Study. Pediatrics,128(3). doi:10.1542/peds.2010-2825d

Schweitzer, J., James, C., Jenkins, W., Reiff, M. I., \& Stein, M. T. (2016). Acute Agitation and SelfInjury in a 5-Year Old with Autism. Journal of Developmental \& Behavioral Pediatrics,37(7), 592-594. doi:10.1097/dbp.0000000000000324

Simrén, M., Barbara, G., Flint, H. J., Spiegel, B. M., Spiller, R. C., Vanner, S., Zoetendal, E. G. (2012). Intestinal microbiota in functional bowel disorders: A Rome foundation report. Gut,62(1), 159176. doi:10.1136/gutjnl-2012-302167

Srikantha, P., \& Mohajeri, M. H. (2018). Microbiota-Gut-Brain-Axis and Autism Spectrum Disorders. doi:10.20944/preprints201811.0171.v1

Strati, F., Cavalieri, D., Albanese, D., Felice, C. D., Donati, C., Hayek, J., Filippo, C. D. (2017). New evidences on the altered gut microbiota in autism spectrum disorders. Microbiome,5(1). doi:10.1186/s40168-017-0242-1

Valicenti-Mcdermott, M., Mcvicar, K., Rapin, I., Wershil, B. K., Cohen, H., \& Shinnar, S. (2006). Frequency of Gastrointestinal Symptoms in Children with Autistic Spectrum Disorders and Association with Family History of Autoimmune Disease. Journal of Developmental \& Behavioral Pediatrics,27(Supplement 2). doi:10.1097/00004703-200604002-00011

Wakefield, A. J., Murch, S. H., Anthony, A., Linnell, J., Casson, D. M., Malik, M., Walker-Smith, J. A. (1998). Ileal lymphoid nodular hyperplasia, non-specific colitis and regressive behavioural disorder: A new syndrome? Journal of Pediatric Gastroenterology \& Nutrition, 25, 47. doi:10.1097/00005176-199700002-00048 (Retraction published February 6, 2010, Lancet, 375. p. 9713)

Williams, B. L., Hornig, M., Buie, T., Bauman, M. L., Paik, M. C., Wick, I., Lipkin, W. I. (2011). Impaired Carbohydrate Digestion and Transport and Mucosal Dysbiosis in the Intestines of Children with Autism and Gastrointestinal Disturbances. PLoS ONE,6(9). doi:10.1371/journal.pone.0024585 


\section{Appendix A}

\section{Assessment Tools}

\section{Employing the Gastrointestinal Symptom Rating Scale (GSRS) in ASD}

The GSRS has been used to assess the effectiveness of microbiota transfer therapy in participants with ASD (Kang et al., 2017). The reliability and validity of the GSRS was evaluated in a six-country study in individuals with dyspepsia (Kulich et al., 2008). The internal consistency reliability of the GSRS was $0.43-0.87$. The test-retest reliability of the GSRS was $0.36-0.75$. The questionnaire clearly differentiated health status based on severity and frequency of symptoms.

The version of the GSRS employed in this study was from McMillan's Headache Research Report. The principal investigator utilized a modified four-point Likert-type scale (McMillan, 2001). This author contacted McMillan and asked for the reliability and validity scores for his modified GSRS. He was unable to provide the needed information. He directed the author to articles that employ the seven-point Likert scale rather than the four-point Likert scale employed in his project. The scale was further modified by a clinical expert in the ASD population. Excluded were questions related to heartburn, sucking sensations in the epigastrium, and borborygmus since they were not relevant in the ASD population.

\section{Employing the Bristol Stool Chart (BSC) in ASD}

The BSC overall had good reliability ( $\kappa=0.72$ ), but with only moderate reliability for Types 2 $(63 \%, \kappa=0.57)$ and $3(62 \%, \kappa=0.55)$ (Blake, Raker, \& Whelan, 2016). The BSC has good validity and intra-rater reliability.

\section{Autism Diagnostic Observational Schedule (ADOS)-1 and ADOS-2}

The ADOS- 2 is a revision of the ADOS- 1 . The second edition contains a toddler module, revised protocols, new comparison score, and updated algorithms. However, the ADOS-2 is administered and coded the same as the ADOS-1. The diagnosis of ASD is based on scoring above the cut off for modules one to four. In the research setting, the ADOS- 1 and ADOS-2 had good interpersonal objectivity and diagnostic accuracy (Kamp et al., 2018). The sensitivity of the ADOS-1 was 0.917 and the specificity was 0.472 (deBilt et al., 2004). 


\section{Appendix B}

\section{Correlations Among Gastrointestinal Symptomatology in ASD}

\begin{tabular}{|c|c|c|c|c|c|c|c|c|c|c|c|c|}
\hline Variable & 1 & 2 & 3 & 4 & 5 & 6 & 7 & 8 & 9 & 10 & 11 & 12 \\
\hline \multicolumn{13}{|l|}{ 1. Abdominal } \\
\hline Pain & 1 & $.445^{* *}$ & 0.249 & $.447 * *$ & 0.102 & $.396^{*}$ & 0.069 & -0.264 & 0.139 & -0.002 & -0.005 & 0.178 \\
\hline \multicolumn{13}{|l|}{ 2. Acid } \\
\hline Regurgitation & $.445^{* *}$ & 1 & $.435^{*}$ & $.483 * *$ & 0.305 & 0.086 & 0.145 & 0.053 & 0.335 & 0.045 & 0.056 & 0.112 \\
\hline \multicolumn{13}{|l|}{ 3. Nausea and } \\
\hline Vomiting & 0.249 & $.435^{*}$ & 1 & $.751 * *$ & 0.338 & 0.212 & -0.03 & -0.111 & 0.332 & 0.085 & 0.18 & $.636^{* *}$ \\
\hline \multicolumn{13}{|l|}{ 4. Abdominal } \\
\hline Distension & $.447 * *$ & $.483 * *$ & $.751 * *$ & 1 & $.493^{* *}$ & 0.265 & 0.141 & -0.249 & 0.232 & 0.134 & 0.047 & $.391 *$ \\
\hline 5. Eructation & 0.102 & 0.305 & 0.338 & $.493 * *$ & 1 & 0.31 & $.494 * *$ & -0.239 & -0.148 & -0.048 & -0.079 & 0.261 \\
\hline 6. Increased Flatus & $.396^{*}$ & 0.086 & 0.212 & 0.265 & 0.31 & 1 & 0.216 & -0.097 & 0.034 & 0.087 & 0.074 & $.481^{* *}$ \\
\hline \multicolumn{13}{|l|}{ 7. Decreased } \\
\hline Passage of Stools & 0.069 & 0.145 & -0.03 & 0.141 & $.494 * *$ & 0.216 & 1 & -0.294 & -0.325 & 0.15 & -0.15 & 0.217 \\
\hline \multicolumn{13}{|l|}{ 8. Increased } \\
\hline Passage of Stools & -0.264 & 0.053 & -0.111 & -0.249 & -0.239 & -0.097 & -0.294 & 1 & $.468 * *$ & -0.244 & 0.004 & -0.081 \\
\hline 9. Loose Stools & 0.139 & 0.335 & 0.332 & 0.232 & -0.148 & 0.034 & -0.325 & $.468^{* *}$ & 1 & -0.309 & -0.127 & 0.023 \\
\hline 10. Hard Stools & -0.002 & 0.045 & 0.085 & 0.134 & -0.048 & 0.087 & 0.15 & -0.244 & -0.309 & 1 & $.511 * *$ & 0.175 \\
\hline \multicolumn{13}{|l|}{ 11. Urgent Need } \\
\hline for Defecation & -0.005 & 0.056 & 0.18 & 0.047 & -0.079 & 0.074 & -0.15 & 0.004 & -0.127 & $.511^{* *}$ & 1 & 0.274 \\
\hline \multicolumn{13}{|l|}{ 12. Feeling of } \\
\hline \multicolumn{13}{|l|}{ Incomplete } \\
\hline Evacuation & 0.178 & 0.112 & $.636^{* *}$ & $.391 *$ & 0.261 & $.481 * *$ & 0.217 & -0.081 & 0.023 & 0.175 & 0.274 & 1 \\
\hline
\end{tabular}

Note. ** Correlation is significant at the 0.01 (2-tailed). * Correlation is significant at the 0.05 level (2-tailed).

Spearman rho correlation interpretation is 00-.19 "very weak", .20-.39 "weak", .40-.59 "moderate", .60-.79 "strong", .80-1.0 "very strong." 